\title{
Who is the cool kid? Biomarkers to help treat newborn brain injury
}

\author{
Niamh Denihan
}

Department of Paediatrics and Child Health, UCC

"To think is to practise brain chemistry" (Deepack Chopra)

\section{The fight for oxygen}

Imagine the birth of a child and you might picture a scene from a movie. A frantic sequence of events culminating in that last big push when the camera focuses on the faces of overjoyed, yet overwhelmed parents. Within seconds it is all over, calmness descends as the newborn child is safely placed in the arms of its loving family. Unfortunately child birth is not always like the movies. In fact, 20 in every 1000 infants can have complications during delivery and need resuscitation after birth. Instead of the typical 'movie scene' birth scenario, the experience can be terrifying and distressing for parents. Delivering the baby is now an emergency and a main concern for the doctor is that the baby might be deprived of oxygen. A world of uncertainty has suddenly been opened.

Oxygen deprivation is serious because it can interrupt the blood supply to the baby's brain causing it to swell. If this is prolonged, brain cells may die and the infant faces the threat of brain damage. In medical terms this brain injury is called hypoxic ischeamic encephalopathy or HIE. In Ireland and the United Kingdom, HIE is the third most common cause of newborn death, while globally it is thought to cause over one million deaths each year. Tragically, even survivors of the injury cause grave concern because they are at risk of debilitating lifelong neurological impairments like cerebral palsy, seizures, developmental delay or learning and behavioural problems. All of these are a major burden for the child, parents and siblings and will severely impact on their quality of life.

Thankfully, over the past decade international clinical trials have uncovered an innovative treatment which reduces the severity of brain injury sustained, and importantly improves the chances of a normal neurological outcome for the infant. This treatment is called therapeutic hypothermia or 'cooling'.

'Hypothermia Cure: Cooling infants to battle brain damage' The Wall Street Journal

The newborn suffering with HIE is wrapped in a cooling blanket for a number of days, as shown in figure 1 , lowering the baby's core body temperature to $33-34^{\circ} \mathrm{C}$ from the typical norm of $37^{\circ} \mathrm{C}$. Cooling has the unique ability to slow down bodily functions. In 
HIE it works by slowing down the death of brain cells that can occur for days after the initial injury. Minimizing cell death is critical for improving the prospect of a life free from neurological disability.

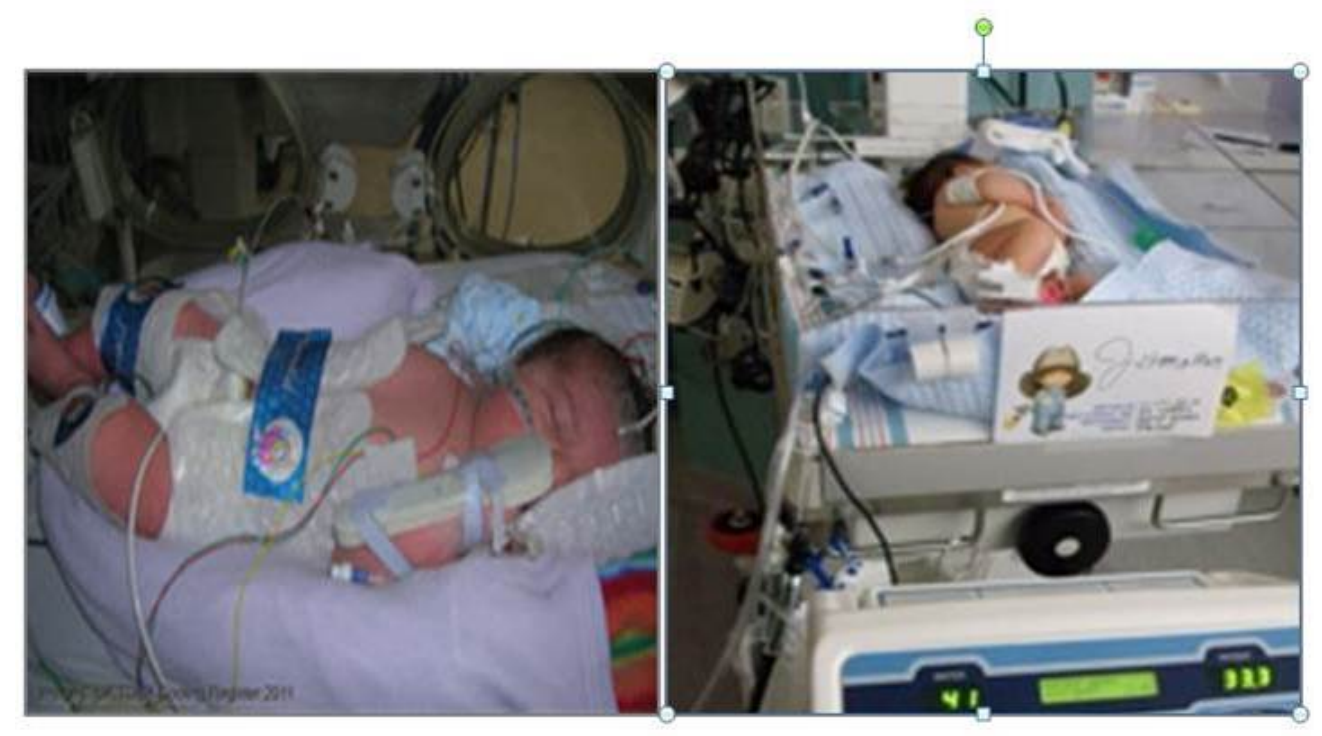

Figure 1: Two infants with HIE wrapped in cooling blankets to undergo the therapeutic hypothermia treatment. Pictures sourced from The UK TOBY Cooling Register () and The Children's Hospital Central California (https://www.childrenscentralcal.org/PressRoom/HospitalNews/Pages/CoolingBlanket.aspx). Reprinted with permission.

\section{To cool or not to cool?}

Cooling has gained increasing popularity and is now the first line of care for newborns at risk of brain injury, but it is not the perfect solution. Researchers have found that most of the permanent cell death and damage to the brain occurs approximately 6-48 hours after birth. This means it is critical to start cooling within the first 6 hours of birth before the brain damage is irreversible. Cooling is essential for these newborns, but hypothermia itself comes with its own health risks. The most common side effects of cooling are low heart rate and an increased use of sedatives to control the infants shivering. Therefore, international consensus agrees that it is only suitable to cool infants at risk of a considerable (moderate to severe) brain injury.

These two factors are greatly hindering our ability to effectively cool infants who need it most. Even in today's sophisticated intensive care units it remains very difficult to assess the extent of a brain injury soon after birth. In fact, it can take up to 24 hours to really determine the severity of the injury sustained, meaning that the critical 6 hour window to treat by cooling can be missed. This pushes the need for early and accurate prediction of brain injuries to the forefront of neonatal science. What use is this innovative and effective treatment, if we don't know who would benefit from it? We are in a race. Each second 


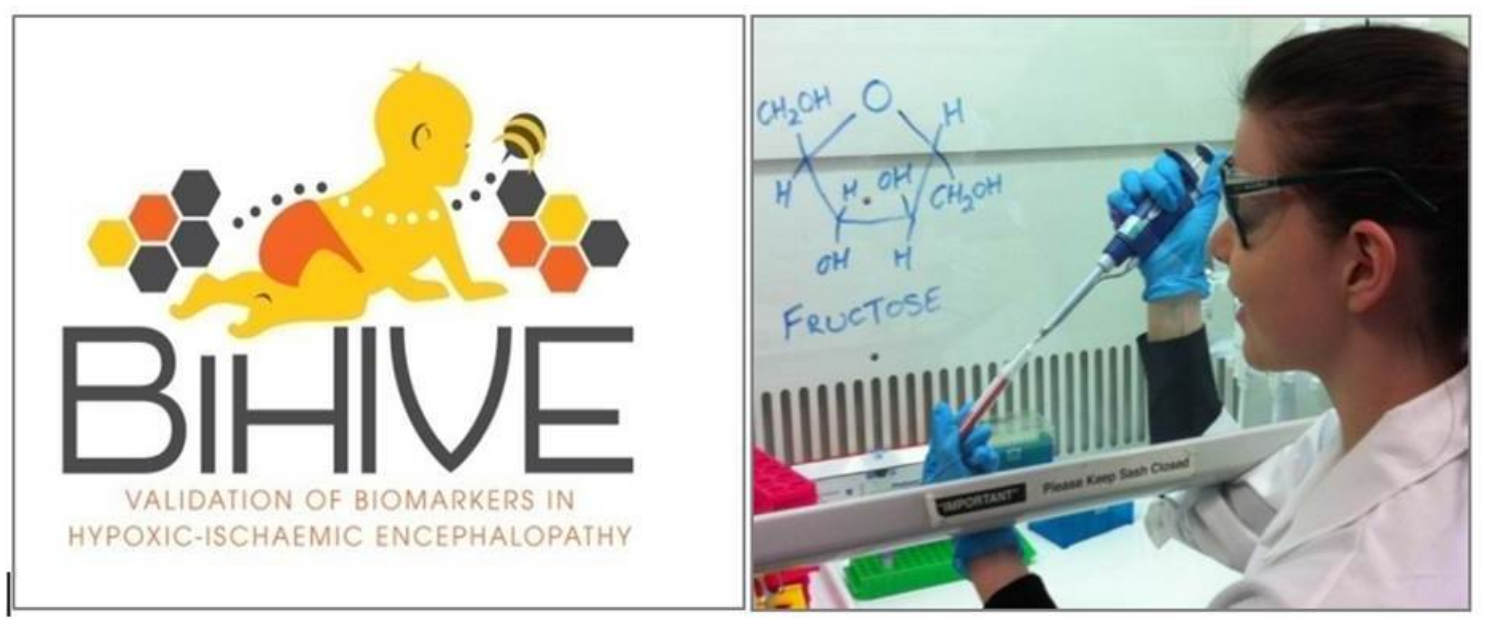

Figure 2: The BIHIVE study logo sourced from www.medscinet.net/BIHIVE/ and metabolomic experiments in action. Image: Niamh Denihan.

wasted is an open invitation for tragedy to strike. My $\mathrm{PhD}$ will tackle this problem head on.

\section{The BIHIVE Study}

What if HIE instantly alters the biochemical makeup of the baby's blood? Could these biochemicals act as markers left behind by the injury? Could these markers tell us how severe the damage is? $\mathrm{My} \mathrm{PhD}$ is based on the premise that these assumptions are true. My aim is to find biochemical markers in infants' blood after they have been deprived of oxygen during birth, which will quickly and accurately identify babies at risk of brain injury. I am a vital cog of a unique research team working on The BIHIVE Study (The Biomarkers in Hypoxic Ischaemic Encephalopathy Study), see logo in figure 2. Our ultimate goal is to create a simple blood test for doctors to use within minutes of birth, which will inform them if a baby is at risk of significant HIE and needs cooling.

I am part of a $24 / 7$ on call team on the front line at Cork University Maternity Hospital (CUMH) that collects blood samples from the umbilical cords of all babies who've had complications during delivery. These samples are carefully stored in dedicated freezers within 3 hours of birth, so that they remain perfectly intact until the time comes to analyse them. The infant's brain activity is monitored closely over the first few days of life using electroencephalography (EEG) which detects abnormal activity or seizures and brain imaging (MRI) to look for structural damage to the brain. The EEG and MRI provide us with a wealth of information, particularly on the severity of brain injury. I will use this information in combination with a state of the art chemistry technique called metabolomics, which allows me to analyse the biochemicals in each infant blood sample. 


\section{Metabolomics: a promising technology}

Metabolomics is fast becoming a popular experimental technique used in science and medicine to study living organisms. It is an experimental technique which allows me to measure the levels of a large number of small chemicals (called metabolites) that are present in blood, like natural sugars and fats, see figure 2. Through these measurements I can create a unique pattern or fingerprint based on that blood sample. This fingerprint of metabolites should give us more information about the 'health' of an organism and in HIE we hope it will quickly diagnose the infants at risk of severe brain injury.

Already the BIHIVE study has published the first ever papers which look at the metabolite fingerprint of infants who were deprived of oxygen during birth and those who developed HIE. Our results demonstrated a difference in the metabolite pattern of infants with HIE compared to healthy infants who had normal births. But more importantly this metabolite pattern could predict the severity of HIE and performed better than the inadequate blood tests that doctors currently use. Our results are not yet $100 \%$ perfect and more work is needed to repeat and validate our finding, before this metabolite pattern can transition from the laboratory bench into a cot-side diagnostic test.

\section{From the lab-bench to the bedside}

Human blood potentially contains thousands of metabolites, but our experiments to date have only focused on a few hundred. In the next stage of my research I will use a chemistry tool called mass spectroscopy to conduct a broad measurement of all metabolites in the infant blood samples. This powerful tool will provide me with a clearer picture of the biochemical makeup of the blood which may improve the effectiveness of our metabolite pattern to diagnose HIE. A large amount of data will be generated by this experiment so I will work closely with statistical and computer experts to analyse it.

The main concerns with this work are that alternate factors may be influencing the metabolomic pattern or that we are finding a pattern purely by chance. Labour is an extremely complex biological process and many factors involved in labour could influence metabolites. For example, the mother's health, how long labour lasted and the type of delivery, could be influencing the patterns that we see. To eliminate this concern I will examine the mother and infant medical notes using statistical tests, to ensure the patterns that we are finding are unrelated to factors in labour. To minimise the risk that we are finding metabolite patterns by chance, I will repeat our previous experiments with samples from a new and separate group of infants. These infants are being enrolled into phase two of the BIHIVE study over the next two years with recruitment currently taking place in CUMH and Karolinska Maternity Hospital, Sweden. We expect the same metabolite pattern to be altered in these infants with HIE, this will confirm our findings are 'real'. To further 
cement our results the last step will be to repeat the experiments in an animal model of HIE. I will collaborate with a research group in Australia who collect and store blood samples from piglets with HIE. As mentioned, HIE is very unpredictable and difficult to detect as the injury occurs sometime around birth, but exactly when is impossible to estimate. Animal models will allow us to control the timing of the injury and to detect exactly when the metabolomic pattern is altered.

Today's modern neonatal intensive care units have made huge progress in keeping newborns who suffered from HIE alive. But the rate of brain injury and long term damage has remained unchanged for the past 20 years. The ultimate goal of this work is to create a useful bedside test with significant and real health benefits for children, families and society as a whole. Identifying infants at risk quickly after birth is essential in getting the maximum benefit from cooling and reducing the neurological injury a child sustains. Even a slight reduction to the effects of HIE can benefit their long term health and quality of life immeasurably. The metabolomic pattern altered in these infants at birth is potentially the key to unlocking the secrets behind this tragic injury and the ability to measure these effects with a simple blood test is tremendously exciting.

I would like to thank my supervisors Dr Deirdre Murray, Prof Geraldine Boylan and The BIHIVE Study team, as well as; the infants, parents and clinical staff who participate in The BIHIVE Study. Many thanks to Molecular Medicine Ireland who fund my PhD project as part of the Clinical \& Translational Research Scholars Programme. 